Bond University

Research Repository

\title{
Focus Group on Social Virtual Reality in Social Virtual Reality: Effects on Emotion and Self- Awareness
}

Manyuru, Patricia; Dobbins, Chelsea; Matthews, Benjamin; Baumann, Oliver; Dey, Arindam

Published in:

2021 IEEE International Symposium on Mixed and Augmented Reality Adjunct (ISMAR-Adjunct)

DOI:

10.1109/ISMAR-Adjunct54149.2021.00099

Licence:

Other

Link to output in Bond University research repository.

Recommended citation(APA):

Manyuru, P., Dobbins, C., Matthews, B., Baumann, O., \& Dey, A. (2021). Focus Group on Social Virtual Reality in Social Virtual Reality: Effects on Emotion and Self-Awareness. In 2021 IEEE International Symposium on Mixed and Augmented Reality Adjunct (ISMAR-Adjunct) (pp. 437-438). IEEE Computer Society. https://doi.org/10.1109/ISMAR-Adjunct54149.2021.00099

\section{General rights}

Copyright and moral rights for the publications made accessible in the public portal are retained by the authors and/or other copyright owners and it is a condition of accessing publications that users recognise and abide by the legal requirements associated with these rights.

For more information, or if you believe that this document breaches copyright, please contact the Bond University research repository coordinator. 


\title{
Focus Group on Social Virtual Reality in Social Virtual Reality: Effects on Emotion and Self-Awareness
}

\author{
Patricia Manyuru ${ }^{1 *}$, Chelsea Dobbins ${ }^{1+}$, Benjamin Matthews ${ }^{1 \ddagger}$, Oliver Baumann ${ }^{2 \S}$, Arindam \\ Dey ${ }^{19}$,
}

\author{
${ }^{1}$ University of Queensland \\ ${ }^{2}$ Bond University
}

*p.manyuru@uq.net.au; †c.m.dobbins@uq.edu.au; łmatthews@uq.edu.au;

§obaumann@bond.edu.au; ๆe-mail: a.dey@uq.edu.au

\begin{abstract}
Social Virtual Reality (VR) platforms enable multiple users to be present together in the same virtual environment (VE) and interact with each other in this space. These platforms are used in different application areas including teaching and learning, conferences, and meetings. To improve the engagement, safety, and overall positive experience in such platforms it is important to understand the effect they have on users' emotional states and self-awareness while being in the VE. In this work, we present a focus group study where we discussed users' opinions about social VR and we ran the focus group in a social VR platform created in Hubs by Mozilla. Our primary goal was to investigate users' emotional states and self-awareness while using this platform. We measured these effects using positive and negative affect schedule (PANAS) and Self-Assessment Questionnaire (SAQ). The experiment involved 12 adult participants who were volunteers from around the world with previous experience of VR.
\end{abstract}




\section{INTRODUCTION}

Online social behaviors are primarily reliant on human interactions in the real world [5]. Virtual Reality (VR) can also enable online social communication and these type of VR interfaces are termed as Social VR interfaces where more than one users can collocate and communicate in real time [7]. Virtual reality has been reviewed as an object of technology [3] and as an application [8]. Over the years, VR has been investigated for collaboration and recently social VR platforms such as AltSpace VR, RecRoom, and Horizon have gained popularity. Bailenson et al. [1] illustrated the utility of using virtual environments (VE) to transform social interaction via behavior and context. Dey et al. [4] investigated the use of physiological feedback to explore emotion and empathy in collaborative VR. However, there are limited investigations that have analysed the emotional and selfawareness effects of social VR particularly in a focus group study. Focus group study is a qualitative data collection method that brings together similar group of people together to discuss a common topic of interest [6]. As social VR setups can connect people remotely in a same environment, it is possible to execute qualitative research with a diverse population. So far there is no example of running a focus group discussion in a social VR setup. This study aims to understand the emotional states and self-awareness of participants in a focus group discussion in VR as this understanding can help researchers and practitioners use this qualitative more effectively in VR research. The main novelty of this work is its innovative methodology, where we used a social virtual environment to run the focus group on topics related to social behaviors in VR.

\section{EXPERIMENT}

The Topic: The primary topics discussed in the focus group were - (a) communication patterns in virtual spaces, (b) social awareness in VR, and (c) adaptive VR interfaces.

The focus group was conducted by a facilitator who used a semistructured protocol, which included information on general aspects of focus groups, an introduction to the subject matter, and an initial exploration of the participants' level of engagement with VR. After this, participants were invited to discuss when they started using VR, how often they use VR and in what capacity they use VR.

Participants: We ran the focus group study with 12 participants ( 5 female) with ages ranging between 21 and 45 years $(M=30.25, S D=6.98)$. To participate in the study, participants were required to have access to their own VR hardware and it was also a requisite to have prior experience in VR. All participants had normal or corrected to normal vision, and normal hearing. Participants were distributed into four matched groups based on age and experience of VR. The native language of all participants was English.

The Virtual Environment, Protocol, \& Equipment: The social VR environment used in this study was designed using Spoke by Mozilla, and was deployed on the platform Hubs by Mozilla. The design of the VE was informed by prior literature, and was substantiated based on a pilot study.

Once in the VE, participants were placed in a studio office, and were asked to take the first two minutes of the interaction to familiarize themselves with the space and how to interact in the VE. After the initial two minutes the were sitting lounge area where the rest of the discussions took place (Figure 1). After the focus group discussions ended, participants completed two questionnaires - Positive and Negative Affect Schedule (PANAS) [9] and Self-Assessment Questionnaire (SAQ) [2]. 
The entire study took on average one hour per participant including the focus group discussions (45 minutes).

A variety of headsets were used including HTC Vive, Oculus Rift, and Oculus Quest that participants had access to. Nine (75\%) of the participants set up their systems for walkable experiences, while the remaining three $(25 \%)$ were set up for seated experiences.

\section{RESULTS \& DISCUSSION}

In this section, we will discuss the results from the data collected though the PANAS and SAQ scales. The detailed results of the focus group are beyond the scope of this poster article.

\subsection{PANAS}

PANAS scale requires participants to respond to 20 (ten positive and ten negative) emotions and feelings. Participants responded on a four-point scale the extent to which they had experienced each feeling and emotion. The positive affect (PA) achieved a mean score of 28.33 (SD = 3.22). This illustrates that the overall positive affect of the experience on the participants can be described as being within the range of "quite a bit positive". By contrast, the negative affect (NA) results achieved a mean score of $11.33(S D=1.7)$. This illustrates that the overall negative affect of the experience on the participants can be described as "slightly/not at all negative."

While analyzing individual emotions and feelings, we noticed that the primary contributors to the overall PA were interested (3.58) and enthusiastic (3.25). The least contributors were strong (1.67) and active (2.17). This demonstrates that participants felt enthusiastic to be taking part in a discussion regarding a topic they are interested in. They did not rank feeling strong or active highly. This was expected because the task did not include any physical activity beyond discussing and it took place in a relaxed and casual setting.

The primary contributors to NA were irritable (1.42) and scared (1.25). In the explanation for this, participants mentioned that encountering technological and technical issues during setup led to feelings of irritation. Further, they indicated that they felt mildly stressed when their connection dropped because they felt like they would be left out of the discussion. The least contributors were hostile and afraid, with both scoring 1 on average. Similar to the reason given for the low-ranking PA mood states, it was expected that feelings of being 'hostile' and 'afraid' would score low because the discussion took place in a calm setting; there were also no sudden changes in the environment.

\subsection{SAQ}

The SAQ questionnaire consisted of six self-evaluation questions, which were selected to assess user perceptions of themselves, other participants, and the SVR environment. The questions were rated on a four-point Likert Scale, which included the options from very slightly/minimal (1) to very much/detailed (4). The questions asked in the questionnaire were: (1) Prior to this session, how much experience have you had in VR environments?; (2) To what extent do you feel like you interacted with the other participants during the discussion?; (3) To what extent did other participants influence your mood or emotional state during the discussion?; (4) How compelling was each of the following aspects of the experience: anonymity; novelty; social tendency; technology? (5) How inadequate was each of the following aspects of the experience: time; technology setup; non-adaptive VE; other users? (6) What one word can you use to sum up your level of social awareness in the VR environment? 
As it can be seen in Figure 2, more than half of the participants (58\%) indicated that the most compelling part of the experiment was the social tendency, or the ability to socialize with other participants in immersive VR. An additional $17 \%$ felt most compelled by the ability to remain anonymous, $17 \%$ indicated that they were most compelled by the VR technology, and the remaining $8 \%$ felt most compelled by the novelty of multi-user (social) VR. Participants indicated that the preselected discussion topics normalized the interactions and gave the discussion focus.

\section{@ Positively Influential $\quad$ Negatively Influential}

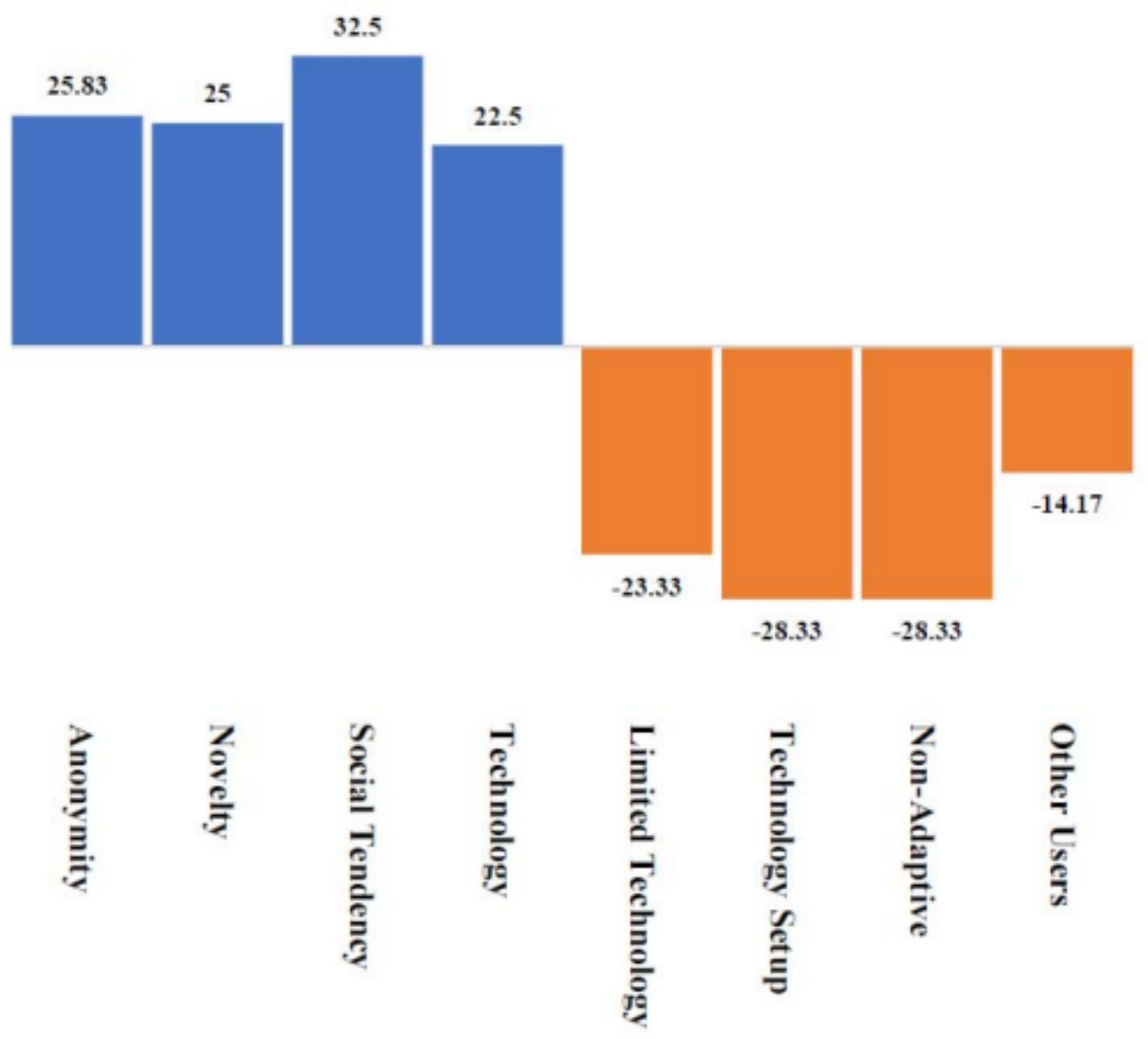

Figure 2: Self-Assessment Questionnaire results.

According to $46 \%$ of the participants, the least compelling aspect was that the environment was not fully interactive as the participants were not able to interact with the elements in the environment. The results also indicated that $27 \%$ of the participants felt that their technology set-ups presented issues and required assistance from the host in troubleshooting during the experience.

4 


\section{CONCLUSION}

In this work, we have presented the first focus group study that discussed about social VR in a social VR environment. Our results showed that in general the participants had more positive affect than negative affect and they felt enthusiastic and interested in the focus group. This indicates that social VR environments can be used to run focus group studies. However, the main limitation of this work is the use of different types of VR headsets which led to slightly different experiences and different technical challenges. As future works will focus on thematic analysis of the focus group discussion, use symmetric social VR setups, and include users who are not already experienced with VR.

\section{REFERENCES}

[1] J. N. Bailenson, N. Yee, J. Blascovich, A. C. Beall, N. Lundblad, and M. Jin. The use of immersive virtual reality in the learning sciences: Digital transformations of teachers, students, and social context. The Journal of the Learning Sciences, 17(1):102-141, 2008.

[2] M. Bertrand and S. Bouchard. Applying the technology acceptance model to vr with people who are favorable to its use. Journal of Cyber Therapy \& Rehabilitation, 1(2), 2008.

[3] G. Burdea and P. Coiffet. Virtual reality technology, 2003.

[4] A. Dey, H. Chen, A. Hayati, M. Billinghurst, and R. W. Lindeman. Sharing manipulated heart rate feedback in collaborative virtual environments. In 2019 IEEE International Symposium on Mixed and Augmented Reality (ISMAR), pp. 248-257. IEEE, 2019.

[5] L. Jin, Y. Chen, T. Wang, P. Hui, and A. V. Vasilakos. Understanding user behavior in online social networks: a survey. IEEE communications magazine, 51(9):144-150, 2013.

[6] D. L. Morgan and R. A. Krueger. The focus group guidebook. Sage, 1998.

[7] F. Moustafa and A. Steed. A longitudinal study of small group interaction in social virtual reality. In Proceedings of the 24th ACM Symposium on Virtual Reality Software and Technology, pp. 1-10, 2018.

[8] M. T. Schultheis and A. A. Rizzo. The application of virtual reality technology in rehabilitation. Rehabilitation psychology, 46(3):296, 2001.

[9] D. Watson and L. A. Clark. The panas-x: Manual for the positive and negative affect scheduleexpanded form. 1999. 